\title{
MIND MODEL AND SOCIAL INTELLIGENCE IN HUMAN-MACHINE COLLABORATIVE SYSTEMS
}

\author{
Hiroshi Nakajima, Yasunori Morishima, Ryota Yamada, Scott Brave, Clifford Nass, \\ Masaki Arao, Shigeyasu Kawaji
}

\author{
OMRON Corporation, Kyoto, Japan, International Christian University, Tokyo, Japan \\ OMRON Advanced Systems, Inc., California, U.S.A., Stanford University, California, \\ U.S.A., Stanford University, California, U.S.A., OMRON Corporation, Kyoto, Japan, \\ Kumamoto University, Kumamoto, Japan
}

\begin{abstract}
Even though social intelligence has not been clearly defined yet, consideration of this new type of intelligence should be important for realizing a new generation of human-machine collaborative systems based on human-centered system design policy. In this article, social intelligence and mind model for implementing socially intelligent agents are studied. At first, emotional and affective aspect of social intelligence is mainly discussed because the aspect is considered as more important in contrast with goal-oriented intelligence. Then, implementation of the mind model and its application as an experiment system is discussed with considering the experiment results. Copyright $\odot 2005$ IFAC
\end{abstract}

Keywords: agents, artificial intelligence, interaction, human-machine interface, human-centered design.

\section{INTRODUCTION}

In the information society of today, functionality and operability of machines have become increasingly more complex and complicated. As a result, the problem of overloading human mind and body with those machines has become more serious. In response to these problems, researches in human-machine interaction have lately given a considerable amount of attention to collaboration between humans and machines because there is a potential that it can provide some important functionality to improve machine usability.

For realizing more natural and better interaction between humans and machines, people from technology side tend to regard information richness of media as the most important. But, this idea usually fails in overloading users. As an example of natural media for humans, there is verbal. However, the verbal interaction technology has not provided enough functionality and performance in practical use because of not only speech recognition problem in machine side but also psychological problem in human side.

In order to realize collaborative functionality between humans and machines, authors emphasize that it is necessary to consider two different aspects of the human-machine interaction, which are physical and psychological. The physical aspect is deeply related to the nature of the information exchanged between human and machine. The psychological aspect is deeply connected to humans' internal states, i.e., their feelings, emotion, mood, etc.

Humans are social animal and have their own mind. At a first glance, reconsideration of these facts seems not necessary to us. But, as a result, these important things have been neglected in the design policy of human-machine systems. As in the instance of the verbal interaction system, the physical aspect of interaction is mainly treated for maintaining recognition performance.

Through these discussions, authours argue that it is necessary to build up a human-centered design policy aiming for machine adaptation to human user to realize a new paradigm of human-machine collaborative systems. From the perspective of the human-machine systems, it can be viewed that there exist not just interfaces but also a society between humans and artifacts in which collaboration can take place among the members. While in the previous research much attention has been paid to the interfaces aspect of interactions since they are a more direct element, the more indirect social aspect of interactions should also be considered to be an important element 
because it has a significant influence on human psychology, which is an important part of human-machine interactions.

The objective of this study is to develop smooth and natural human-machine collaborative systems based on the human-centered design concept, especially regarding new type of intelligence - social intelligence.

In this article, mind model is proposed for actualizing social intelligence of agents that embed it themselves. The mind model is used for controlling machine's social behavior for building up a social relationship between humans and machines with a special consideration on social intelligence. In the rest of this paper, social intelligence, mind model and its implementation, and experiment results will be surveyed and discussed.

\section{SOCIAL INTELLIGENCE}

Even though what constitutes social intelligence has not been clearly understood yet, it is hard to ignore this type of intelligence when human-centered design policy should be considered.

At first, a human-machine collaborative system is formalized as a notation (1).

$$
C S=[H, M, I, E, G]
$$

where $H$ :human(s), $M$ :machine(s), $I$ :interaction between $H$ and $M$, E:environment for $H$ and $M$, and $\mathrm{G}$ : goal(s) shared by $H$ and $M$. According to the discussion in previous section, authors will focus on affective and emotional aspects of the interaction $I$. As the prerequisite of it, it is important to treat the environment $E$ as social environment.

\subsection{Rational Behavior and Irrational Behavior}

In the environment $E$ of the notation (1), there are two kinds of behavior, i.e., rational behavior and irrational behavior. According to the present social accepted idea especially in advanced nations, rational behavior is strictly connected with selfish benefits usually achieving economic effects. On the other hand, irrational behavior is considered as emotion and affect basis. That is, this type of behavior has been taken as to contribute nothing to economic effects. Frank pointed out that this intuitive observation is not always correct (Frank, 1988), with explaining following facts. There are many people who run into doing emotional and irrational behavior even though the behavior obviously results in their own disadvantage but other's advantage from the economic point view. Frank also mentioned that this irrational and unselfish behavior results in their benefit.

According to (Frank, 1988), there are two kinds of models for explaining human behavior. The first one is named 'Self-interest model' that is based on economic value criteria. This model hypothesizes that human likes selfish profit such as consumption and leisure; therefore, human affect and emotion such as jealousy, a sense of guilt, anger, honor, love, etc. play no role in gaining profit. On the other side, Frank proposed named 'Commitment model'. According to the model, humans do behavior that is even irrational and opposed to their profit at a glance by committing with themselves on the behavior. In the commitment achievement, emotion and personality that can be observed by other people are effective. That is besides on that emotion and personality are important for behavior selection, in building up social relationship, they are also important to be observed by other people. For instance, people do not tend to sue a person who has a trait of liking sue against such a trifling affair.

\subsection{Social Intelligence and Rational Intelligence}

Katagiri pointed out that social intelligence makes sense especially in the human-machine collaborative systems in contrast with rational intelligence (Katagiri, 2003). 'Rational intelligence' refers to the kind of intelligence that artificial intelligence has traditionally dealt with. According to (Katagiri, 2003), it is goal-oriented intelligence that enables one to find an answer to a question, generate a solution to a problem, and so on. 'Social intelligence' is the type of intelligence that enables one to share information and feelings with others, and behave in such a way which one is accepted as a member of a community. Katagiri listed social emotion as a component of social intelligence. Lewis discriminated between basic emotion such as happy, sad, etc. and social emotion such as honor, jealousy, etc.(Lewis, 2000).

\subsection{Cognitive Appraisal Theory}

Appraisal theories of emotion and most emotion theorists in general agree that emotion is essentially a reaction to events deemed relevant to the needs and goals of an individual (Brave and Nass, 2003). At the most basic level, the achievement of goals leads to happiness, and the failure to achieve goals leads to sadness: this is the current level employed in our system. Various versions of appraisal theory exist which specify in greater detail the critical properties of a goal-relevant event that lead to specific emotions (e.g., frustration, anger, hope, etc.). Goals can take many forms, but Maslow's Hierarchy provides a useful way of categorizing these goals. In his later work, Maslow grouped an individual's basic needs into eight categories (Maslow, 1968):

- Physiological: hunger, thirst, bodily comforts, etc.

- Safety/security: being out of danger

- Social: affiliate with others, be accepted

- Esteem: to achieve, be competent, gain approval and recognition

- Cognitive: to know, to understand, and explore

- Aesthetic: symmetry, order, and beauty

- Self-actualization: to find self-fulfillment and realize one's potential

- Transcendence: to help others find self-fulfillment and realize their potential.

Unlike appraisal theory, current 2-factor theories of emotion (e.g., Lang, 1995) do not focus on what causes emotion, but instead argue that all emotions can be fully described using two dimensions: valence 
(positive/negative) and arousal (low/high).

\subsection{Self-oriented Emotion and Other-oriented Emotion}

Brave employed Empathy to do theoretical survey for defining self-oriented emotion and other-oriented emotion (Brave, 2003). Self-oriented emotion is deeply connected with basic emotion and other-oriented emotion such as empathy is social emotion. Brave extended cognitive appraisal theory for explaining other-oriented emotion based on Batson's Empathy-altruism hypothesis (Batson, 1991). The basic idea is that when people care about another person (i.e., value their welfare), people may take on some of their goals as if they were our own. Such "adopted" goals may now lead to emotion just as any of our own personal goals. When humans react emotionally to adopted goals, it is called 'empathy.'

\subsection{Personality}

People recognize and respond to personalities of other individuals that they interact with. Personality is often used to describe and predict an emotional state and behaviors of an individual. It is considered to be a more stable trait of a person's subjective state. In other words, it does not change dramatically in a short period of time depending on the changes in the environment though it may change over time in the long run. In psychology, so called 'Big Five' is known to characterize some major attributes of personality (Heinström, 2003). They are openness, conscientiousness, extraversion, agreeableness, and emotional stability.

Reeves and Nass assert that friendliness (friendly vs. unfriendly) and dominance (dominant vs. submissive) are two major attributes of personality, especially that of mediated agents (Reeves and Nass, 1996).

\section{MIND MODEL AND ITS IMPLEMENTATION}

Authors have developed a mind model called the Mind and Consciousness Model (MaC model) (Ushida, et al., 1998). Authors claim that agents with a mind model will be required to realize smooth communication and comfortable control in the human and machine collaboration system because agents must have the feature of autonomy, flexibility, and social orientation (Alonso, 2002).

\subsection{Conceptual Design of Mind Model}

Fig.1 shows the conceptual architecture of the $\mathrm{MaC}$ model. The model is based on the cognitive appraisal theory that has discussed previous section. Authors have extended the one of the famous cognitive model (Ortony, et al., 1988) based on the appraisal theory in two areas to develop the MaC model. The first one is to add an information path from the emotional process to the cognitive process. By this extension, cognitive process will have the capability of highly sophisticated processing mechanism with a high-level problem solution task such as recognition, decision-making, planning, etc. The second is a couple of layered information process loops. The first loop is the reflex, in which the reflex component processes the data from sensors to control the actuators, in the way of a rough but speedy process method. The second is the deliberative loop that is corresponding to a richer information processing based on the cognitive appraisal theory. Deliberative process is accurate but slow. The combination of these loops tends to give agents more flexible and intelligent capabilities.

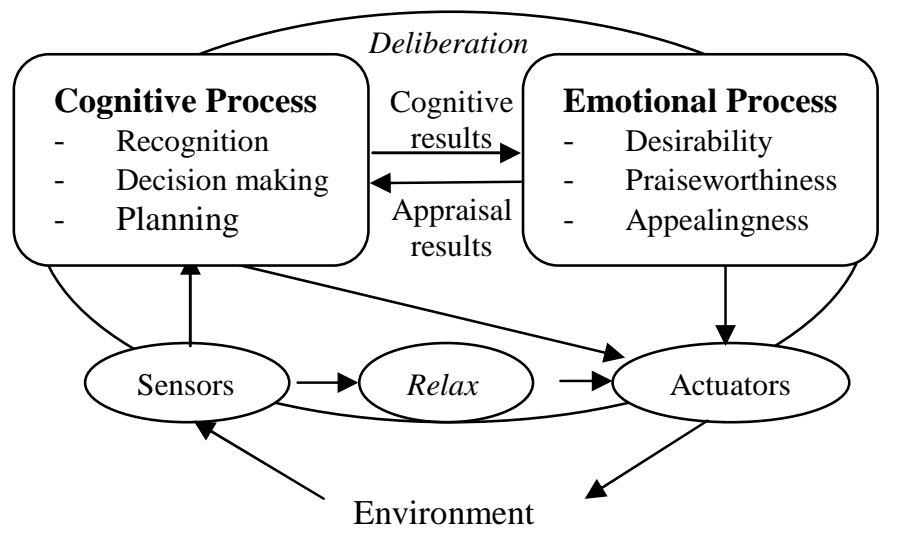

Fig. 1. The conceptual architecture of MaC model

Emotion plays a significant part in the MaC model and also strongly related to social behavior. The theory of the Urge system proposed by Toda (Toda, 1992) has been employed to implement our emotion engine as the role of Emotional Process of the MaC model in Fig. 1. According to the theory, the occurrence mechanism of emotion can be logically explained in terms of situation and emotional factors. The emotion engine calculates six basic emotions. Explicit components for the emotion types have been defined according to (Ekman, 1992). Each component of the emotional factors and the basic emotions has the degree of activation level. Fuzzy inference has been employed to calculate the degree of the each emotional factor from the situation. Then, each activation value is calculated. Activation level of the basic emotions is used to select a behavior such as facial expression and domain specific action.

\subsection{Parameters of Mind Model for Controlling Social Behaviors}

The MaC model has several parameters to control social behaviors of agents that embed it into themselves. The typical parameters for controlling social behaviors are shown in the Table 1.

By these parameter settings, the $\mathrm{MaC}$ model can give certain personality of an agent embedding the model in itself. For example, when the values for 'Desire' and 'Persistency' of the goals are high, the personality of the agent will be controlled as to be strong dominant. According to the personality design, the threshold values for emotional expression and action selection can be adequately set. For example, low threshold value for happiness may result in connecting friendly personality. 
Table 1 Parameters of MaC model for Controlling

$$
\text { Social Behaviors }
$$

\begin{tabular}{|c|c|c|}
\hline Parameter Items & \multirow{2}{*}{\multicolumn{2}{|c|}{$\begin{array}{c}\text { Parameter Values } \\
\text { Desire Deoree }\end{array}$}} \\
\hline Innate Goals & & \\
\hline Empirical Goals & \multicolumn{2}{|c|}{ Persistency Degree } \\
\hline \multirow{6}{*}{$\begin{array}{l}\text { Emotional } \\
\text { Expression }\end{array}$} & \multirow{6}{*}{$\begin{array}{l}\text { Threshold } \\
\text { Value for } \\
\text { Each } \\
\text { Emotion }\end{array}$} & Happiness \\
\hline & & Anger \\
\hline & & Sadness \\
\hline & & Fear \\
\hline & & Disgust \\
\hline & & Surprise \\
\hline \multirow{4}{*}{ Action Selection } & \multirow{4}{*}{$\begin{array}{l}\text { Threshold } \\
\text { Value for } \\
\text { Each Action }\end{array}$} & Action \#1 \\
\hline & & Action \#2 \\
\hline & & $\ldots$ \\
\hline & & Action \#n \\
\hline
\end{tabular}

\section{EXPERIMENT AND RESULTS}

In this section, an experiment system that employs a socially intelligent agent will be presented. A mind model is embedded into the agent to control its social responses. This mind model is simpler than $\mathrm{MaC}$ model because of considering experiment cost but has the same effects and influences as $\mathrm{MaC}$ model on controlling social responses. The application is computer-assisted learning. Education, as traditionally interpreted through classroom-based school systems, is a social activity in many respects, particularly in that learning is mediated and influenced by the content and manner of social interactions between teachers and students, and among students. Authors argue that affective aspects of those social interactions can be utilized to make learning activities more effective and beneficial.

In recent years, there has been a major shift in the paradigm of computer-supported learning. The research efforts on learning systems have traditionally been centered on individualized environments where a single user interacts with the computer system, which often serves as a teacher or a tutor. The new trend is an emphasis on collaborative learning environments (Goodman, et al., 1998; Kasai and Okamoto, 1999). There are some benefits for learning as follows. One is what is often called 'learning by teaching,' in which one can learn given knowledge by explaining it to another learner. The other benefit is often called 'leaning by observation' or 'learning by imitation,' in which one can learn given knowledge by observing another learner working on problem solving, teaching other learners, and so on. While in these approaches to collaborative learning, learning takes place in the interactions between the learners, the kind of intelligence these approaches are primarily concerned with is knowledge-based, goal-oriented, and rational, and thus social intelligence might only be utilized as a side effect. In contrast, our approach attempts to make use of affective and social intelligence in a more direct manner.

\subsection{Experiment System}

A virtual classroom has been developed as a social environment. The learning supporting system incorporates social intelligence for human-machine interaction. A notable characteristic of our system is the introduction of a co-learner agent as a social actor in the virtual environment.

As shown in Fig.2, our learning system employs a classroom metaphor. There are three cartoon characters in the classroom: a teacher agent, a co-learner agent, and an avatar for the human learner. Thus, there occur interactions between the teacher and a learner and interactions between the learners. Among these interactions, authors focus on the interaction between a human learner as an avatar and a co-learner agent. The learning content of the application is English idiom learning.

The co-learner agent is equipped with the mind model that controls their behaviors including emotional expressions.

The mind model uses the personality profile information of each agent to generate behaviors of the agent. The agents and the avatar use facial expressions (see Fig.3.) and text output to communicate with each other. A blackboard is available for the teacher agent and the students to present learning materials.

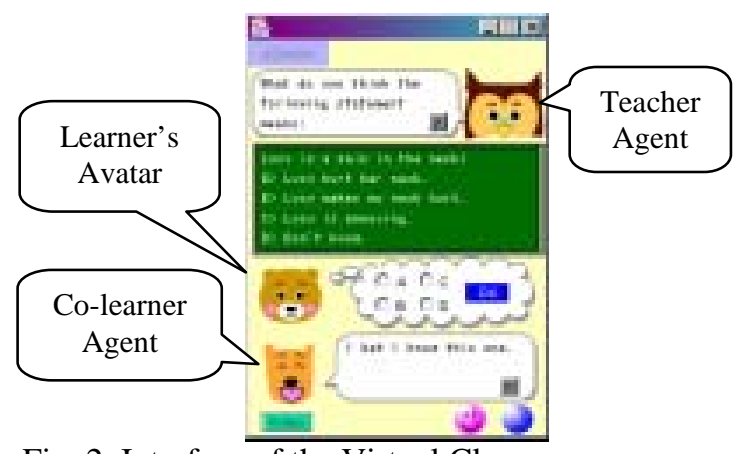

Fig. 2. Interface of the Virtual Classroom

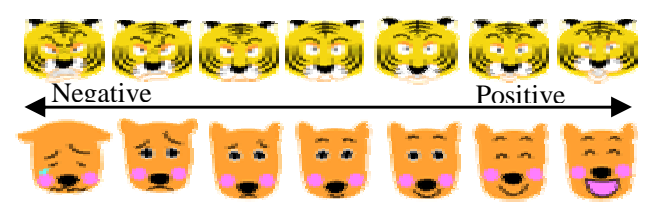

Fig. 3. Facial Expressions

\subsection{Experiment}

Psychological experiments was conducted to examine the effect of the mind model based social intelligence in the collaborative learning system described above.

Design; Regarding of the effect of mind model based social intelligence, three versions of the application were prepared for the experiment:

No co-learner agent (No Agent, NA); On the interface, there were only the teacher agent and the avatar.

Co-learner agent without social intelligence(Agent with No Mind Model, ANMM); On the interface, there were the teacher agent, the co-learner agent, and the avatar. The co-learner agent did not have mind model. It did not display any emotional or social responses, having a 'porker face.'

Co-learner agent with social intelligence(Agent with Mind Model, AMM); On the interface, there were the teacher agent, the co-learner agent, and the avatar. The co-learner agent has mind model to control expression of his social responses. It had friendly and mildly dominant (or, confident) personality, and displayed 
emotional and social responses depending on the human learner's performance.

Method; Participants are 77 undergraduate students at International Christian University (ICU) participated in the experiment. All participants were native speakers of Japanese learning English.

Procedure: The experiment was conducted in ICU's language lab, where all the participants participated in the experimental session together. Each of the participants was randomly assigned to one of the three conditions. After the self-enrolment procedure, the participants had the experimental session of learning English idiomatic expression using the application. The format of learning was a sequence of question, response, feedback, and explanation.

The teacher agent either called on the avatar of the human learner or the co-learner agent to answer. The teacher agent then gave correct or incorrect as feedback. If the human learner had answered, the co-learner agent made a social response to the situation and vice versa.

After the session was over, the participants responded to a questionnaire that was presented on the computer. The questionnaire included questions concerning impressions on the application, the co-learner agent, and user experiences. A short quiz was followed to measure the participants' performance. The experiment took approximately an hour.

\subsection{Results and Discussion}

Quiz Results and Self-assessment of Learning; The effectiveness of the application was first examined based on the results of the quiz, which are shown by Table 2 The participants in the two agent conditions ( $A M M$ and $A N M M$ ) marked higher scores than those in the NA condition. These results seem to indicate that the application was more effective for learning when there was a co-learner agent. Furthermore, the no-response rate was much lower with the AMM than with the other conditions. This seems to suggest that the participants felt more reciprocally motivated to answer the questions when they worked with the co-learner agent with social intelligence.

Table 2 Quiz Results (Ratio to the total number of

\begin{tabular}{ccc} 
& \multicolumn{2}{c}{ responses) } \\
Conditions & Correct & No Answer \\
\hline NA & .51 & .27 \\
ANMM & .60 & .22 \\
AMM & .69 & .07 \\
\hline
\end{tabular}

The questionnaire assessed how much (in a 10-point scale) the participants felt they had learned the materials. Table 3 shows the results.

Table 3 Self-Assessment of Idiom

\begin{tabular}{cccc} 
& $\boldsymbol{N A}$ & $\boldsymbol{A N M M}$ & $\boldsymbol{A M M}$ \\
\hline Mean & 3.86 & 4.96 & 5.28 \\
SD & 2.08 & 2.34 & 2.31 \\
\hline
\end{tabular}

The statistical analysis showed that the participants' self-assessment of their learning was significantly higher for the co-learner agent conditions (AMM and $A N M M)$ than for the $N A$ condition $[F(1,76)=5.32, p$
$<$.05]. This pattern of results indicates that the participants had a more positive impression about their experience and the content of learning when they worked with the co-learner agent than when there was no co-learner agent. Combining the results of the quiz performance, it seems to be legitimate to infer that the data supported the hypothesis that the application is more effective when there is a co-learner agent working with the user than when there was no co-learner agent.

However, the difference between the two co-learner agent conditions ( $A M M$ and $A N M M)$ did not turn out to be significant $[F(1,76)=.26$, n.s.]. To examine effects of the $A M M$ condition, further analyses were performed on other aspects of the participants' learning experiences and impressions.

Evaluation of Co-learner Agent; At first, it was tested whether the participants' impression (evaluation in a 10 -points scale) varied depending on the presence or absence of social responses. The analysis showed that the $A M M$ condition marked significantly higher scores than the $A N M M$ condition in the following evaluation items:

- $\quad$ Cooperativeness $[F(1,51)=13.82, p<.01]$

- $\quad$ Trustworthiness $[F(1,51)=8.56, p<.01]$

- $\quad$ Feels warm $[F(1,51)=6.6 .5, p<.02]$

These results showed that social intelligence and behaviors generated by it had more positive influence on the impressions of the co-learner agent.

Evaluation of Leaning System; As stated above, the purpose of this study was to investigate the effect that the affective aspects of social intelligence may have on the usefulness and impression of the learning system. The question that should be asked is how those impressions and evaluations that the participants had about the agents influenced the evaluation of the application as a whole.

Before broaching into this question, there is one factor that should be taken into consideration. The analysis indicated that under both of the $A M M$ condition and the $A N M M$ condition, the impressions on the application was dependent on how the participant was attracted by the agent $[F(1,51)=12.16, p<.01]$. This means that regardless of the presence of social intelligence, liking varied from person to person. In other words, some individuals liked the agent without social responses more than others and some liked the agent with social responses less than others, depending on their personal preferences. Thus, it is necessary to control for this personal preference effect in analyzing the effect of the social intelligence.

The analysis of covariance was performed with the personal preference on the agent as a covariate. The result revealed that the main effect of the social intelligence controlled by the mind model on the evaluation of the application was found in the following items:

- $\quad$ Ease of use $[F(1,51)=6.11, p<.02]$

- Satisfaction (Would recommend it to others) $[F(1,51)=4.82, p<.04]$

- $\quad$ Pleasantness in learning (Not frustrated) $[F(1,51)=6.62, p<.02]$

- $\quad$ Supportivenss $[F(1,51)=3.28, .05<p<.08]$ 
For these evaluation items, the $A M M$ condition marked higher scores than the $A N M M$. These results seem to suggest that the social intelligence and the social responses (i.e., friendly and confident) that are generated by the mind model had positive impact on the impressions on the learning system as a whole.

Finally, some may argue that it was not the model-based affective responses that impacted on the participants.

The analysis of covariance with the system impression as a covariate showed that the participants felt significantly less fun to work with the system in the $A N M M$ condition not only than the $A M M$ condition but also than the $N A$ condition $\quad[F(1,76)=$ $5.25, p<.03]$. This result seems to indicate that the lack of appropriate affective responses of an agent is worse than the absence of an agent.

In summary, the results of the experiment provided support for the following assertions:

- The co-learner agent contributed to the effectiveness of the learning system.

- The users had more positive impressions about the usefulness and the application and learning experience when the co-learner agent displayed some social responses with personality and emotions.

It should be noted here that the co-learner agent in the experiment system did not provide any explicit assistance for the learner such as giving clues and showing answers. It only made some limited social responses such as praising, encouragement, and comforting. Yet, the participant's evaluation on the usefulness of the learning system was influenced by such an agent.

\section{SUMMARIES AND CONCLUSIONS}

In this article, the mind model and social intelligence of an agent and its application to a collaborative learning environment have been discussed. The agent embeds the mind model into itself to generate social responses. Authors argued that socially appropriate affective behaviors would provide a new dimension for collaborative systems.

Even if the co-leaner agent lacks any intelligence to support the human learner in a direct manner such as giving hints, providing explanations, and guiding him/her through a problem, the leaner left supported and displayed more active involvement in the learning. Thus, the experiment result agrees that the social intelligence has a potential to enhance humanmachine collaboration, if not applying rational intelligence. According to the theories of Media Equation (Reeves and Nass, 1996), people treat computers, television, and new media like real people and places. One implication that this study may make is that one way to build agents including real robots that act supportively is to implement the ability to make social responses appropriate to the situation and to the human user's internal state, as is partially done by our mind model for realizing social intelligence.

\section{REFERENCES}

Alonso, E. (2002). AI and Agents - State of the Art. In: Intelligent Systems, FALL, 2002, pp 25-29.

Batson, C. D. (1991). The altruism question: Toward a social-psychological answer. Hillsdale, NJ: Lawrence Erlbaum Associates.

Brave, S. (2003) Agents that care: Investigating the effects of orientation of emotion exhibited by an embodied computer agent. Unpublished doctoral dissertation. Stanford University.

Brave, S., and Nass, C. (2003). Emotion in humancomputer interaction. In J. Jacko \& A. Sears (Eds.), The Human-Computer Interaction Handbook (pp. 81-93). Mahwah, NJ: Lawrence Erlbaum Associates.

Ekman, P.(1992). An Argument for Basic Emotions, In: Basic Emotions edited by N. Stein , and K. Oatley, Lawrence Erlbaum, Hove, U.K., pp. 169-200,

Goodman, B., Soller, A., Linton, F., and Gaimari, R. (1998) Encouraging student reflection and articulation using a learning companion. International Journal of Artificial Intelligence in Education, 9(3-4).

Heinström, J.( 2003). Five personality dimensions and their influence on information behaviour. Information Research, Vol. 9 No. 1.

Kasai, T., \& Okamoto, T. (1999). Kasoteki kyocho kankyo-niokeru ejento-kan koraboreshon. (Collaboration among agents in a virtual collaborative learning environment.) Johoshori Gakkai Ronbunshi, 40 (11), 3934-3945.

Katagiri, Y. (2003). Robot no shakai-teki chinou (Robots with social intelligence.) In:IPSJ Magazine Vol.44, No.12, pp.1233-1238.

Lang, P.J. (1995). The emotion probe. American Psychologist, 50(5), 372-385.

Lewis, M. (2000). The Emergence of Emotions, chapter 17, pp.265-280, Guilford Press.

Maslow, A.H. (1968). Toward a Psychology of Being. New York: D. Van Nostrand Company.

Ortony, A., Clore, G., and Collins, A. (1988). The Cognitive Structure of Emotions, Cambridge University Press, New York.

Reeves, B., and Nass, C. (1996). The Media Equation: How People Treat Computers, Television, and New Media Like Real People and Places, CSLI publications and Cambridge University Press, New York.

Toda, M.(1992). Kanjo (Emotion - The Innate Adaptive Software System That Drives human Beings, )University of Tokyo Press, Tokyo.

Ushida, H., Hirayama, Y., and Nakajima, H. (1998). Emotion Model for Life-like Agent and Its Evaluation. In: Proceedings of AAAI-98: Fifth National Conference on Artificial Intelligence, Madison, pp. 62-69. 